\title{
Three patients with defects in interferon gamma receptor signaling: a challenging diagnosis
}

\author{
Zijun Zhou ${ }^{1}$, Iris Hollink ${ }^{1}$, Arjan Bouman ${ }^{1}$, Mirthe Lourens ${ }^{1}$, Rik Brooimans ${ }^{1}$, Tjakko van \\ Ham $^{1}$, Peter Fraaij ${ }^{1}$, Annemarie van Rossum ${ }^{2}$, Eline Zijtregtop ${ }^{2}$, Willem Dik ${ }^{1}$, Virgil van \\ Dalm$^{1}$, Martin van Hagen ${ }^{3}$, Hanna Ijspeert ${ }^{1}$, and Clementien Vermont ${ }^{1}$ \\ ${ }^{1}$ Erasmus MC \\ ${ }^{2}$ Sophia Children's Hospital of the Erasmus University Medical Center in Rotterdam \\ ${ }^{3}$ Erasmus MC, University Medical Center Rotterdam
}

October 31, 2021

\begin{abstract}
Background: Defects in IFN-gamma receptor (IFN- $\gamma \mathrm{R}$ ) signaling via STAT1 leads to susceptibility to infection by otherwise weak pathogenic mycobacteria, resulting in mendelian susceptibility to mycobacterial disease. We identified three patients presented with disseminated mycobacterial infections caused by M. avium, M. persicum or M. bovis BCG respectively. Wholeexome sequencing (WES) was used as the first line diagnostic approach, however in all patients additional analysis was crucial to make the definite diagnosis. Method: WES, SNP array and long range PCR were performed to identify the genetic defects. Expression of IFNGR1, STAT1, CD64, SOCS1 and phosphorylation of STAT1 were determined after stimulation with IFN- $\alpha$ or IFN- $\gamma$. Results: In Patient 1, only one heterozygous variant p.(Val63Gly) in the IFNGR1 gene was identified by WES. Additional genetic analysis identified a second complex Alu-insertion in IFNGR1. Patient 2 was compound heterozygous for the null p.(Val68Lysfs*6) variant and the hypomorphic p.(Ile37Thr) variant in IFNGR1. In Patient 3 a novel variant in the STAT1 gene p.(Asn460Ile) was identified. Patients 1 and 2 had reduced expression of IFN- $\gamma$ R1. All patients had reduced phosphorylation of STAT1 and absent induction of SOCS1 after IFN- $\gamma$ stimulation. While STAT1 phosphorylation was normal after IFN- $\alpha$ stimulation in Patient 1 and 2, and mildly reduced in Patient 3. Conclusion: We conclude that functional assays are crucial to assess the extent of IFN- $\gamma \mathrm{R}$ signaling defects when new combinations of bi-allelic or non-conclusive genetic variants are found, which is important in the determination of clinical treatment.
\end{abstract}

Three patients with defects in interferon gamma receptor signaling: a challenging diagnosis

Zijun Zhou, ${ }^{1,2,6}$ Iris H.I.M. Hollink, ${ }^{3,6}$ Arjan Bouman, ${ }^{3,6}$ Mirthe S. Lourens, ${ }^{1,6}$ Rik A. Brooimans, ${ }^{1,6}$ Tjakko J. van Ham, ${ }^{3}$ Pieter L.A.Fraaij, ${ }^{4,6}$ Annemarie M.C. van Rossum ${ }^{4,6}$ Eline A.M. Zijtregtop, ${ }^{5}$ Willem A. Dik, ${ }^{1,6}$ Virgil A.S.H. Dalm, ${ }^{1,2,6}$ P. Martin van Hagen, ${ }^{1,2,6}$ Hanna IJspeert, ${ }^{1,6 \#}$ Clementien. L. Vermont ${ }^{4,6 \# *}$

${ }^{1}$ Erasmus MC, University Medical Center Rotterdam, Laboratory Medical Immunology, department of Immunology, the Netherlands

${ }^{2}$ Erasmus MC, University Medical Center Rotterdam, department of Internal Medicine, Division of Clinical Immunology, the Netherlands

${ }^{3}$ Erasmus MC, University Medical Center Rotterdam, department of Clinical Genetics, the Netherlands

${ }^{4}$ Erasmus MC, University Medical Center Rotterdam-Sophia Children's Hospital, department of Pediatrics, Division of Pediatric Infectious Disease and Immunology, the Netherlands 
${ }^{5}$ Erasmus MC, University Medical Center Rotterdam-Sophia Children's Hospital, department of Pediatrics, Division of Pediatric Hemato-oncology, the Netherlands

${ }^{6}$ Erasmus MC, University Medical Center Rotterdam, Academic Center for Rare Immunological Diseases (RIDC), the Netherlands

\# these authors contributed equally

Running title: IFN- $\gamma$ R signaling defects in MSMD patients

Correspondence:

Dr. Clementien L.Vermont

Erasmus MC University Medical Center-Sophia Children's Hospital

PO Box 2060, 3000 CB

Rotterdam the Netherlands

Email: c.vermont@erasmusmc.nl

Main text: 1248 words

Number of tables and figures: 2

Supplemental data: 1 Figure and methods

\section{Disclosure of conflicts of interest}

The authors have no conflict of interest.

\section{Financial Support}

The authors received no financial support for the research, authorship or publication of this article.

\section{Main text:}

To the editor,

Mendelian susceptibility to mycobacterial disease (MSMD) is a rare inherited disorder characterized by infections with weakly virulent mycobacteria in otherwise healthy individuals. ${ }^{1}$ MSMD is caused by genetic defects in genes coding for proteins in the IL- $12 / \mathrm{IFN}-\gamma$ signaling pathway. ${ }^{2}$ IFN- $\gamma \mathrm{R} 1$ deficiency is a common cause of MSMD. ${ }^{1}$ The pathogenic variants inIFNGR1 can be either autosomal recessive (OMIM \#209950) or dominant (OMIM \#615978), ${ }^{3}$ resulting in complete or partial functional defects. ${ }^{4}$ Lack of surface expression leads to a complete loss of function, while expression of hypomorphic IFN- $\gamma \mathrm{R} 1$ can lead to either partial loss or complete loss of function. Complete loss of function in IFN- $\gamma \mathrm{R}$ signaling results in severe mycobacterial infections in early childhood and poor survival, while variants with partial loss of function may result in a milder clinical phenotype that manifests later in childhood. A general approach in diagnosing patients with MSMD has been proposed in literature using flow cytometry, lymphocyte immunophenotyping and sequencing of genes known to be involved in mycobacterial host immunity. ${ }^{4,5}$ However, since recent advances in genomic analysis technologies, a genotype-first approach for patients with a suspicion of an inborn error of immunity has currently become a more typical approach. ${ }^{6}$ While having the advantage of analyzing multiple genes simultaneously, some variants can be missed and the interpretation of the significance of novel variants or new combination of hypomorphic variants can be a diagnostic challenge. Here, we describe three patients with MSMD with novel or new combinations of hypomorphic variants in which functional assays were crucial to determine the extent of the interferon gamma receptor signaling defects.

Patient 1 is a 6-year old girl who was admitted to our pediatric ward with progressive cough, fever and tachypnea. Her medical history was remarkable with more than 20 hospital admissions for bronchial hyperreactivity, lower respiratory tract infections and a coarctectomy at the age of 2 . Imaging showed mediastinal 
and hilar lymphadenopathy, a large right upper lobe mass and lymph nodes compressing the trachea (Supplementary Fig S1A). An ultrasound showed three focal lesions in the spleen and hepatomegaly. Because of her severely compromised airway, steroids were given, improving intraluminal tracheal diameter. Histological examination on the lymph node ruled out malignant lymphoma. Sputum, stomach fluid, lymph node biopsy and bone marrow culture revealed Mycobacterium avium. Plasma IFN- $\gamma$ level on admission was elevated $(311 \mathrm{pg} / \mathrm{ml})$. She was treated with azithromycin, rifampicin and ethambutol. Amikacine was added for the first 2 months. She recovered after a year of treatment. Patient 2 is a 7 -year old boy was admitted with unexplained and recurrent low-grade fever, neck and leg pain, vasculitis-like skin lesions and polydipsia for several months. He had a medical history with recurrent bronchial hyperreactivity, recurrent parotitis, parechovirus-associated radiculitis and cluster headache. A brain MRI, CT scans of thorax and vertebrae, total body X-ray studies and abdominal ultrasound showed a hypophysitis leading to panhypopituarism, several bone lesions in skull, vertebrae and hip, hilar lymphadenopathy and a single lesion in the spleen. Tuberculin skin test showed induration of $18 \mathrm{~mm}$. Cultures of a bone lesion and sputum grew Mycobacterium persicum. Serum IFN- $\gamma$ levels were undetectable. He started treatment with rifampicin, isoniazid and ethambutol as well as hydrocortisone, levothyroxine and desmopressin for his panhypopituarism, and has improved very well. Patient 3 is a 1-year old girl with a swollen right upper arm and axillary lymphadenopathy. She had a BCG scar that looked inflamed. Her medical history showed bronchial hyperactivity and eczema. A CT scan showed multiple active hilar and axillary lymph nodes, a splenic lesion and osteolytic lesions of her right humerus, two ribs and a vertebral lesion in L2. Pathology of a bone biopsy from the right humerus showed lesions compatible with Langerhans cell histiocytosis (LCH). Treatment for multifocal $\mathrm{LCH}$ was started using prednisone and vinblastine. The bone lesions showed improvement but new skin lesions appeared at the site of the BCG scar, head and back. Repeated CT scans showed multiple bone lesions in scapulae, ribs and progression of hilar and axillary lymphadenopathy. Skin biopsies of the lesions showed granulomatous infiltration and grew Mycobacterium bovis BCG strain. The previous diagnosis of $\mathrm{LCH}$ was re-evaluated and a new diagnosis of disseminated BCG-itis was made. Treatment with rifampicin and isoniazid resulted in rapid improvement on skin and bone lesions as well as on lymphadenopathy.

A diagnostic whole exome sequencing (WES)-based PID gene panel analysis was used as first line diagnostic approach. In Patient 1 one heterozygous pathogenic variant in the IFNGR1 gene NM_000416.2(IFNGR1):c.188T > G, p.(Val63Gly) was identified. However, no second variant was detected. Interestingly, only theIFNGR1 transcript with the p.(Val63Gly) variant was identified with RNA expression analysis, and a SNP array showed decreased binding of one probe in exon 3 of IFNGR1 . Long range PCR using primers surrounding exon 3 showed a heterozygous insertion. Sequence analysis revealed that this insertion concerned a complex insertion of 136 nucleotides of the right arm and the poly-A tail of an AluY transposable element combined with a 4-nucleotide duplication in exon 3: NM_000416.2(IFNGR1):c.210_211ins[136;A[?];207_210] (Fig. 1AB). Patient 2 was compound heterozygous for a known pathogenic variant NM_000416.2(IFNGR1):c.373+1G>T, p.(?) and variant NM_000416.2(IFNGR1):c.110T>C, p.(Ile37Thr), classified as probable pathogenic (Fig. 1AB). In Patient 3 a novel variant in theSTAT1 gene: NM_007315.3(STAT1):c.1379A > T, p.(Asn460Ile) was identified (Fig. 1C). Parental investigation showed this was a de novo variant (Fig. 1A).

Functional analysis was performed to determine the effect on the IFN- $\gamma / \mathrm{STAT} 1$ signaling pathway. The surface expression of IFN- $\gamma \mathrm{R} 1$ on monocytes was severely reduced in Patient 1 and Patient 2 , while the expression was normal or even elevated in Patient 3 (Fig. 2A). In Patient 1 and 2, the expression of STAT1 was normal (Supplementary Fig. S1B), while the phosphorylation of STAT1 was severely reduced in response to stimulation IFN- $\gamma$, but normal upon IFN- $\alpha$ stimulation (Fig 2BC). Furthermore, the upregulation of the IFN-gamma regulated geneSOCS1, was absent after stimulation with IFN- $\gamma$ (Fig. 2D), and normal upon IFN- $\alpha$ stimulation (Fig. 2E). In line with that, membrane expression of the IFN-gamma regulated gene CD64 was not increased after IFN- $\gamma$ stimulation in Patient 2. These functional data suggest that Patient 1 and 2 have a defect in IFN-gamma signaling. For Patient 1 the life-threatening presentation of disease in combination with the results of the functional analyses led to consideration of allogeneic hematopoietic stem cell transplantation as a treatment option, for which patient and her parents are counselled. 
The STAT1 gene variant p.(Asn460Ile) in Patient 3, is located in the DNA binding domain of the STAT1 protein. Studies on the crystal structure of STAT1 showed that Asn460 is important for DNA binding since it is the only amino acid that directly interacts via hydrogen bonds to DNA, implying that this amino acid is crucial for STAT1 function. ${ }^{7,8}$ In Patient 3, STAT1 protein expression was not affected (Supplementary Fig. S1B), but the phosphorylation of STAT1 was slightly decreased (Fig. 2B). Moreover, it seemed to be delayed upon IFN- $\gamma$ and IFN- $\alpha$ stimulation in monocytes compared to HCs (Fig. 2C). Expression of SOCS1 seemed normal after $0.5 \mathrm{~h}$ of stimulation with IFN- $\gamma$, but was reduced after 1 and 2 hours of stimulation (Fig. 2D). Together, these functional data and the fact that the variant is de novo strongly suggest that Patient 3 has an AD STAT1 deficiency.

In conclusion, patients presenting with disseminated mycobacterial infections in early or late childhood with bi-allelic or non-conclusive genetic variants in genes involved in mycobacterial immunity need to be phenotyped by functional assays of the IFN- $\gamma / \mathrm{STAT} 1$ signaling pathway in monocytes. These assays are crucial in assessing the extent of IFN- $\gamma$ pathway signaling defects and in clinical decision making.

Key Words : mendelian susceptibility to mycobacterial disease, IFN- $\gamma$ receptor, STAT1, mycobacterial infection, SOCS1

Zijun Zhou, ${ }^{1,2,6}$ Iris H.I.M. Hollink, ${ }^{3,6}$ Arjan Bouman, ${ }^{3,6}$ Mirthe S. Lourens, ${ }^{1,6}$ Rik A. Brooimans, ${ }^{1,6}$ Tjakko J. van Ham, ${ }^{3}$ Pieter L.A.Fraaij, ${ }^{4,6}$ Annemarie M.C. van Rossum,${ }^{4,6}$ Eline A.M. Zijtregtop, ${ }^{5}$ Willem A. Dik, ${ }^{1,6}$ Virgil A.S.H. Dalm, ${ }^{1,2,6}$ P. Martin van Hagen,,${ }^{1,2,6}$ Hanna IJspeert, ${ }^{1,6 \#}$ Clementien. L. Vermont ${ }^{4,6 \# *}$

${ }^{1}$ Erasmus MC, University Medical Center Rotterdam, Laboratory Medical Immunology, department of Immunology, the Netherlands

${ }^{2}$ Erasmus MC, University Medical Center Rotterdam, department of Internal Medicine, Division of Clinical Immunology, the Netherlands

${ }^{3}$ Erasmus MC, University Medical Center Rotterdam, department of Clinical Genetics, the Netherlands

${ }^{4}$ Erasmus MC, University Medical Center Rotterdam-Sophia Children's Hospital, department of Pediatrics, Division of Pediatric Infectious Disease and Immunology, the Netherlands

${ }^{5}$ Erasmus MC, University Medical Center Rotterdam-Sophia Children's Hospital, department of Pediatrics, Division of Pediatric Hemato-oncology, the Netherlands

${ }^{6}$ Erasmus MC, University Medical Center Rotterdam, Academic Center for Rare Immunological Diseases (RIDC), the Netherlands

\# these authors contributed equally

\section{Acknowledgements}

The research for this manuscript was performed within the framework of the Erasmus Postgraduate School Molecular Medicine.

\section{References}

1. Bustamante J, Boisson-Dupuis S, Abel L, Casanova JL. Mendelian susceptibility to mycobacterial disease: genetic, immunological, and clinical features of inborn errors of IFN-gamma immunity. Semin Immunol. 2014;26(6):454-470.

2. Kerner G, Rosain J, Guerin A, et al. Inherited human IFN-gamma deficiency underlies mycobacterial disease. J Clin Invest.2020;130(6):3158-3171.

3. McKusick-Nathans Institute of Genetic Medicine JHU, Baltimore, MD. OMIM. Online Mendelian Inheritance in Man, OMIM@R. Retrieved from https://omim.org/. 2021.

4. van de Vosse E, van Dissel JT. IFN-gammaR1 defects: Mutation update and description of the IFNGR1 variation database. Hum Mutat.2017;38(10):1286-1296. 
5. Wu UI, Holland SM. Host susceptibility to non-tuberculous mycobacterial infections. Lancet Infect Dis. 2015;15(8):968-980.

6. French MA, Tangye SG. The Next Generation of Diagnostic Tests for Primary Immunodeficiency Disorders. The Journal of Infectious Diseases. 2019;221(8):1232-1234.

7. Chen X, Vinkemeier U, Zhao Y, Jeruzalmi D, Darnell JE, Jr., Kuriyan J. Crystal structure of a tyrosine phosphorylated STAT-1 dimer bound to DNA. Cell. 1998;93(5):827-839.

8. Basab Roy ZZ, Gary D. Stormo. Quantitative specificity of STAT1 and several variants. Nucleic Acids Research. 2017;45(14):8199-8207.

TablesNone.

Figure legends

Fig.1 Overview of the genetic variants identified inIFNGR1 and STAT1

Family pedigree of the patients indicating the genetic variant identified the patients (A). Linear presentations of the human IFN- $\gamma \mathrm{R} 1$ (B ) and STAT1 protein $(\mathbf{C})$ indicating the different domains and genetic variants identified in the three patients. SP, signal peptide; TM, transmembrane domain; TAD, transactivation domain. The binding location for JAK1 and STAT1 are indicated with black lines. The numbers underneath the IFN- $\gamma \mathrm{R} 1$ and STAT1 proteins indicate the amino acid numbering. The numbers underneath the AluY transposable element indicate the nucleotide numbering of the AluY.

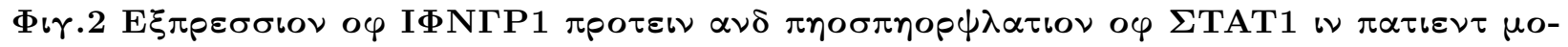

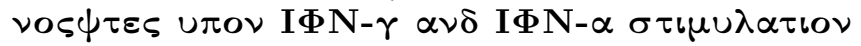

Analysis of IFNGR1 (CD119, clone GIR-208) on patient CD45+CD3-CD14+ monocytes (blue) and healthy control (HC)(red). Patient 1 and Patient 2 showed reduced expression of IFN- $\gamma \mathrm{R} 1$ in monocytes compared to the $\mathrm{HC}(\mathrm{n}=2)$ (A) Flowcytometric analysis of phosphorylated STAT1 (pSTAT1) protein in patient monocytes after stimulation with $60 \mathrm{IU} / \mathrm{ml} \mathrm{IFN}-\gamma$. Patient 1 had no pSTAT1 expression, Patient 2 had severely reduced and Patient 3 had slightly reduced pSTAT1 expression. The median fluorescence intensity (MFI) for two independent experiments are shown in (B ) $(\mathrm{HC} n=2$; Patient 1: $n=2$; Patient 2: $n=2$; Patient 3: $\mathrm{n}=2$ ). MFI of pSTAT1 for two independent experiments in patient monocytes after stimulation with

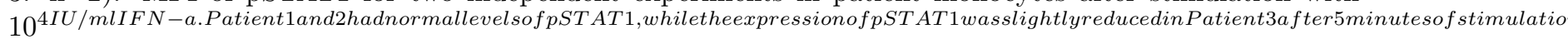


A
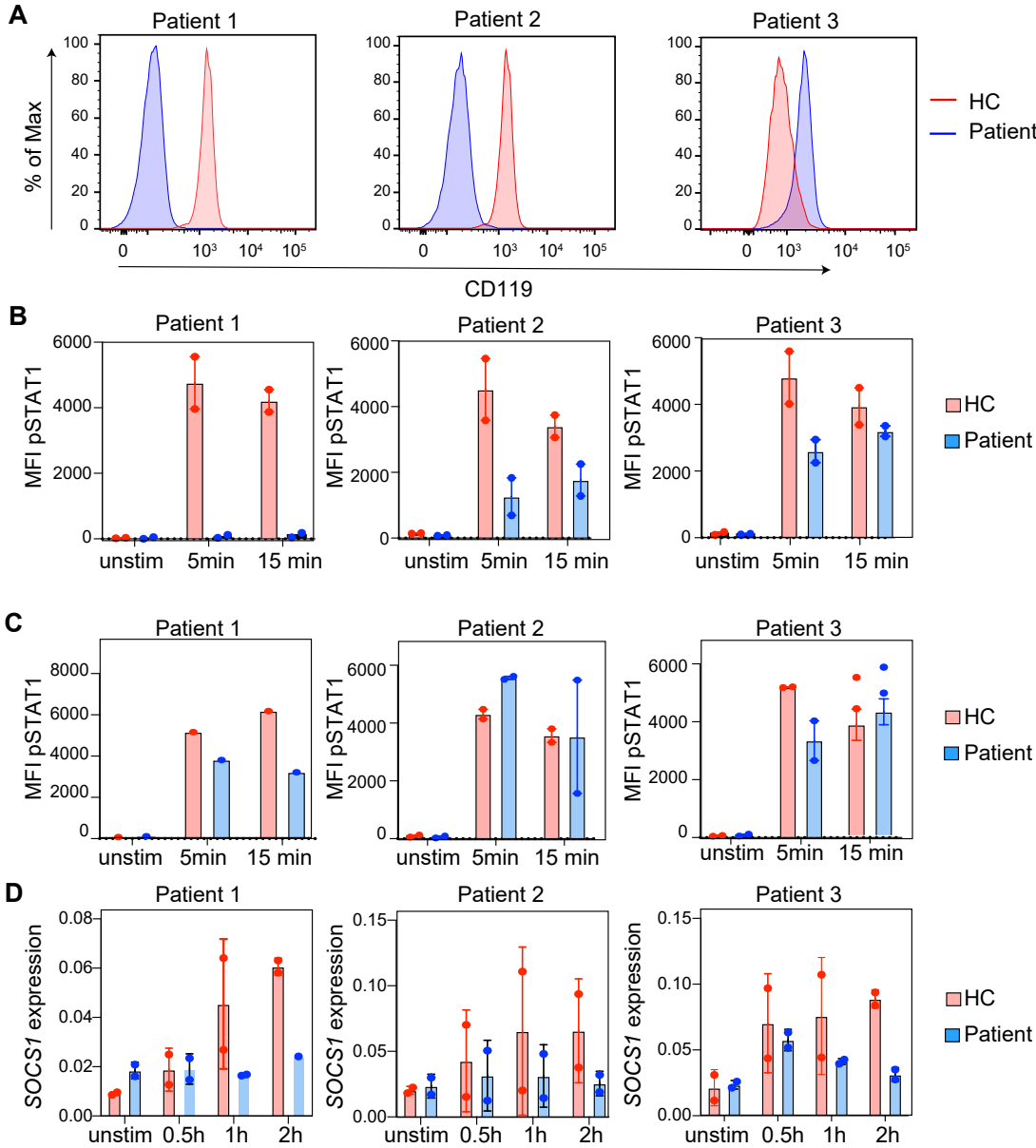

E
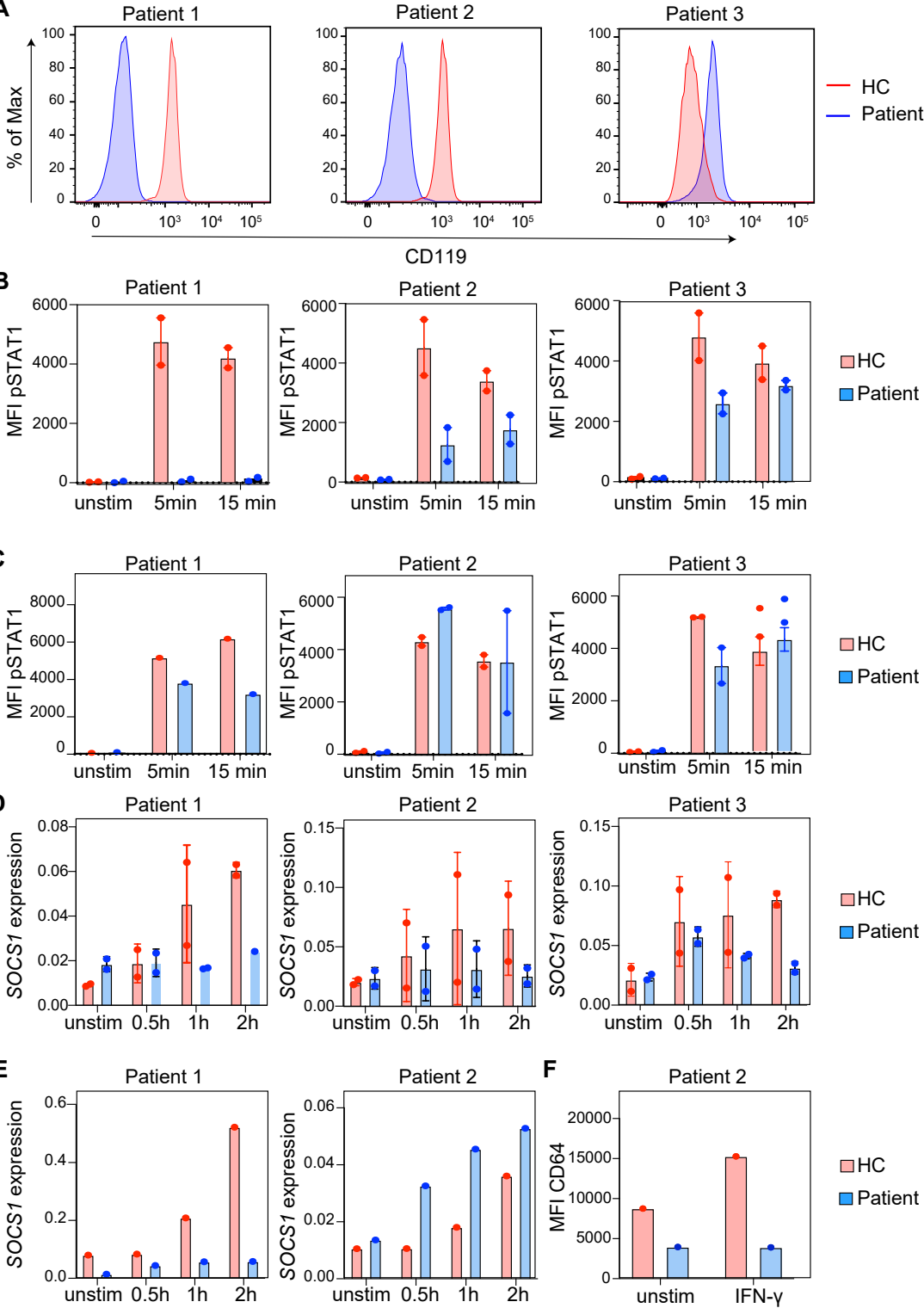

$\square \mathrm{HC}$

$\square$ Patient

$\square \mathrm{HC}$

$\square$ Patient

F Patient 2

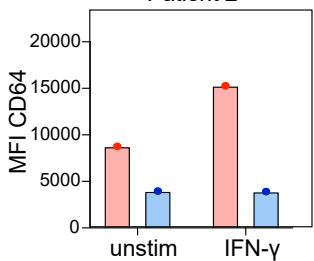

$\square \mathrm{HC}$

$\square$ Patient 